\title{
Distribution and expression of SLC45A2 in the skin of sheep with different coat colors
}

\author{
Haidong Wang', Linli Xue ${ }^{2}$, Yanan $\mathrm{Li}^{1}$, Bingling Zhao ${ }^{1}$, Tianzhi Chen ${ }^{1}$, \\ Ying Liu ${ }^{1}$, Lucheng Chang ${ }^{1}$, Juan Wang ${ }^{3}$
}

${ }^{1}$ College of Animal Science and Veterinary Medicine, Shanxi Agricultural University, Taigu, Shanxi, China

${ }^{2}$ College of Information, Shanxi Agricultural University, Taigu, Shanxi, China

${ }^{3}$ Department of Geriatrics, Tongji Hospital of Shanghai affiliated with Tongji University, Shanghai, China

\begin{abstract}
Introduction. To investigate whether the membrane-associated transporter protein SLC45A2 is differentially expressed in the skin of sheep with different coat colors and to determine its correlation with coat color establishment in sheep.

Material and methods. The expression of SLC45A2 in sheep skin samples with different coat colors was qualitatively and quantitatively analyzed by PCR amplification, RT-PCR, immunohistochemical staining and Western blotting.

Results. A 193-bp SLC45A2 CDS sequence was successfully amplified from sheep skin samples with diverse coat colors. RT-PCR analysis revealed that SLC45A2 mRNA was expressed in all sheep skin samples tested, with relative expression levels of $512.74 \pm 121.51$ in black skin, $143.38 \pm 119.31$ and $1.36 \pm 0.09$ in black dots and white dots of piebald skin, respectively, and $1.02 \pm 0.23$ in white skin $\left(\mathrm{p}<0.01^{* *}\right)$. Positive SLC45A2 protein bands were also detected in all skin samples by Western blot analysis, with relative expression levels of $0.85 \pm$ $\pm 0.17^{* *}$ in black skin, $0.60 \pm 0.05^{* *}$ and $0.34 \pm 0.07$ in black dots and white dots of piebald skin, respectively, and $0.20 \pm 0.05$ in white skin $\left(\mathrm{p}<0.01^{* *}\right)$. Immunohistochemical assays revealed that SLC45A2 was expressed in the hair follicle matrix, the inner and outer root sheath, and the dermal papilla in the skin tissues with different coat colors. These patterns were quantified by optical density (OD) analysis, which yielded relative expression levels of $0.23 \pm 0.11$ in black skin, $0.19 \pm 0.09$ and $0.10 \pm 0.03$ in black dots and white dots of piebald skin, respectively, and $0.08 \pm 0.01$ in white skin $\left(\mathrm{p}<0.05^{*}\right)$.

Conclusion. SLC45A2 is detectably expressed in sheep skin of all coat colors, though at significantly different levels. SLC45A2 may participate in the establishment of coat color by regulating the synthesis and trafficking of melanin. (Folia Histochemica et Cytobiologica 2016, Vol. 54, No. 3, 143-150)
\end{abstract}

Key words: SLC45A2; sheep; coat color; Q-PCR; Western blot; IHC

\section{Introduction}

SLC45A2 was originally identified as an antigen recognized by reactive $\mathrm{T}$ cells in human melanoma

\footnotetext{
Correspondence address: Prof. J. Wang

Department of Geriatrics

Tongji Hospital of Shanghai

Shanghai, 200065 China

tel.: +86-13623541993

e-mail: whd1232123@163.com
}

and was thus named AIM-1 (antigen in melanoma). To distinguish this gene from a serine/threonine kinase, it was subsequently called MATP (membrane-associated transporter protein) before finally being termed SLC45A2. Its expression in melanocytes and melanoma cell lines has been confirmed [1]. SLC45A2 gene is located on human chromosome 5P13.3, with a length of approximately $40 \mathrm{~kb}$. SLC45A2 is a protein consisting of 530 amino acids and 12 transmembrane domains and localizes to the cell membrane of melanosomes, playing the role of a transporter protein 
during the synthesis of melanin [2, 3]. SLC45A2 is a member of the putative transporter family $45 \mathrm{~A}$, which includes four members: SLC45A1, SLC45A2 (MATP), SLC45A3 and SLC45-A4 [4]. SLC45A2 encodes a protein with homology to plant sucrose-H+ symporters, but the transport function of SLC45A2 is not known. SLC45A2 is important for processing and trafficking tyrosinase to melanosomes [5].

Human disorders caused by disruptions in melanin synthesis in melanosomes (and the associated causative genes) include oculocutaneous albinism OCA1 (TYR), OCA2 (P), OCA3 (TRP1) and OCA4 $(M A T P)$ [6]. Researchers have determined that mutations in $S L C 45 A 2$ can abrogate the processing and trafficking of tyrosinase at the Golgi level, leading to oculocutaneous albinism type IV, a low pigment disorder with autosomal recessive inheritance $[3,7,8]$. These mutations not only alter the pigmentation of medaka fish [9], mice [2, 10], horses [11], chicken and quail [12] but are also correlated with abnormal eye and skin color development in humans [13-15]. The membrane-associated transporter protein encoded by the $S L C 45 A 2$ gene has been recognized as a sodium-hydrogen exchanger that functions in melanosomes to regulate the activity of tyrosinase in human melanocytes [16]. Interestingly, Bin et al. found that knockout of $S L C 45 A 2$ significantly reduced the $\mathrm{pH}$ of melanosomes, with further analyses revealing that SLC45A2 affects the activity of tyrosinase by adjusting the $\mathrm{pH}$ of melanosomes and thus plays an important role in the regulation of tyrosinase turnover [17]. Baxter et al. reported that SLC45A2 is expressed in pigment precursor cells during embryonic development and that its protein expression pattern is similar to those of the melanin-producing enzyme dopachrome tautomerase (DCT) and tyrosinase-related protein 1 (TYRP1), suggesting that this gene is like-wise dependent on microphthalmia-associated transcription factor (MITF), as in the retinal pigment epithelium [18]. However, the expression of SLC45A2 in early neural crest-derived melanocytes is similar to that of DCT, but not TYR or TYRP1 [19]. SLC45A2 has thus served as new marker of the migratory melanoblast population, which previously could only be identified by the co-expression of DCT, MITF and KIT.

Our study was initiated to investigate whether the expression and localization of SLC45A2 in sheep skin of different coat colors was directly associated with the pigment level, thereby indicating a role for this protein in regulating the synthesis of melanin and affecting the establishment of coat color. To date, no studies have reported the relative expression levels of SLC45A2 in sheep skin samples of different coat colors.
SLC45A2 expression in sheep skin was measured by multiple methods. By determining the correlation between SLC45A2 and coat color, our results not only provide insight into the mechanism of coat color establishment in mammals but also reveal important genetic information and related experimental evidence for further studies of the coat color forming pathways.

\section{Material and methods}

Animals. Three approximately one-year-old healthy smalltailed Han sheep were randomly selected from breeds with white, black and piebald coat colors (provided by the Hunyuan sheep farm, China). After shaving and then disinfection, three pieces of dorsal skin tissue, approximately $1 \mathrm{~cm}$ across, were collected from each sheep using a skin collector, of which two samples were frozen and stored in liquid nitrogen for total RNA and total protein extraction. The remaining piece of skin was fixed in Bouin's solution to prepare paraffin sections for immunohistochemical staining.

Materials. The main reagents in this study included TRIzol (Invitrogen, Carlsbad, CA, USA), a reverse transcription PCR kit (Takara, Dalian, China), SYBR ${ }^{\circledR}$ Premix Ex TapTM II (Takara, Dalian, China), RIPA lysis buffer (Beyotime Institute of Biotechnology, Shanghai, China), Protein Marker (Thermo Scientific, Grand Island, NY, USA), a SLC45A2 rabbit polyclonal antibody (Santa Cruz Biotechnology, Santa Cruz, CA, USA), a GAPDH rabbit polyclonal antibody (Proteintech Group, Wuhan, China), horseradish peroxidase (HRP)-conjugated goat anti-rabbit IgG (Beijing Com-Win Biotech, Beijng, China), and an ECL Western Blot Kit (Beijing ComWin Biotech).

RNA extraction and cDNA synthesis. Total RNA was extracted from the sheep skin samples by the TRIzol method. The integrity of the obtained RNA sample was preliminarily determined by $1 \%$ agarose gel electrophoresis, and then, the RNA concentration was measured using a nucleic acid/ /protein analyzer. Complementary strand cDNA was synthesized following the instructions of the reverse transcription kit from Takara. The reverse transcription reaction included $1 \mu \mathrm{L}$ of oligo dT primer, $1 \mu \mathrm{L}$ of dNTP mix, $\leq 5 \mu \mathrm{g}$ of total RNA and water to a total volume of $10 \mu \mathrm{L}$. After mixing thoroughly, the reaction was performed at $65^{\circ}$ for $5 \mathrm{~min}$, followed by rapid cooling on ice. Subsequently, $4 \mu \mathrm{L}$ of $5 \times$ PrimeScript II Buffer, $0.5 \mu \mathrm{L}$ of RNase inhibitor, $1 \mu \mathrm{L}$ of Primer Script II RTase and water were added to reach a total volume of $20 \mu \mathrm{L}$. After mixing, the reaction was incubated at $42^{\circ}$ for $45 \mathrm{~min}$ and $70^{\circ}$ for $15 \mathrm{~min}$. After the completion of the reaction, the concentration was measured using a nucleic acid/protein analyzer, and the samples were stored at $-20^{\circ}$ for later use. 
Table 1. Primers used to amplify the target genes and PCR amplification conditions

\begin{tabular}{|l|c|c|c|}
\hline \multirow{2}{*}{ Target gene } & Primer sequence (5'-3') & PCR product size (bp) & Annealing temperature $\left(^{\circ}\right)$ \\
\hline \multirow{2}{*}{ SLC45A2 } & F:ACATTCCCTCACAGCAAGCC & 193 & 60 \\
\cline { 2 - 2 } & R:CTCCTCAATGCCCTCAACAGT & & \\
\hline \multirow{2}{*}{$18 S$ rRNA } & F:GAAGGGCACCACCAGGAGT & 158 & 60 \\
\cline { 2 - 3 } & R:CAGACAAATCACTCCACCAA & & \\
\hline
\end{tabular}

Primers' design. The primers for real-time PCR amplification were designed based on the sheep SLC45A2 sequence in GenBank using Premier 5.0, and the specificity of the primers was preliminarily checked by NCBI. The primers were synthesized by Beijing Genomics Institute (BGI). The primer details are shown in Table 1.

PCR amplification was performed using these specific primers and an appropriate amount of cDNA as the template. The PCR products were detected by $1 \%$ agarose gel electrophoresis: after running at $220 \mathrm{~V}$ for $10 \mathrm{~min}$, the gel image was acquired using an electrophoresis gel imaging system. The purified PCR products were submitted to BGI for bi-directional sequencing.

QRT-PCR amplification. Fluorescence quantitative PCR was performed using the SYBR ${ }^{\circledR}$ Premix Ex TapTM II kit, with each $10-\mu \mathrm{L}$ reaction consisting of $5 \mu \mathrm{L}$ of SYBR ${ }^{\circledR}$ Premix Ex TapTM II $(2 \times), 0.4 \mu \mathrm{L}$ of the forward and reverse primers $(10 \mu \mathrm{M}), 0.2 \mu \mathrm{L}$ of ROX Reference Dye II $(50 \times)$, $\leq 100 \mathrm{ng}$ of cDNA template and $\mathrm{dd}_{2} \mathrm{O}$ to a total volume of $10 \mu \mathrm{L}$. The reaction conditions were as follows: denaturation at $95^{\circ}$ for $10 \mathrm{~s}$, followed by $40-45$ cycles of $95^{\circ}$ for $10 \mathrm{~s}, 60^{\circ}$ for $20 \mathrm{~s}$, and $72^{\circ}$ for $10 \mathrm{~s}$. The internal reference was amplified under the same conditions, and each sample was run with $\geq 3$ technical replicates. After the reaction was complete, quantitation was performed using the $\mathrm{C}_{\mathrm{T}}$ values obtained by the amplification curve. The relative expression levels of SLC45A2 in the black skin, black dots in the piebald skin, white skin, and white dots in the piebald skin were calculated by the $2^{-\Delta \Delta C \mathrm{CT}}$ method: $\Delta \mathrm{C}_{\mathrm{T} \text { target gene }}=\mathrm{C}_{\mathrm{T} \text { target gene }}-\mathrm{C}_{\mathrm{T} \text { reference gene? }}$, $\Delta \Delta \mathrm{C}_{\mathrm{T}}=\Delta \mathrm{C}_{\mathrm{T} \text { black sheep group }}-\Delta \mathrm{C}_{\mathrm{T} \text { white sheep group }}$. The expression of SLC45A2 mRNA was represented as the differences in multiples of $2^{-\triangle \triangle C T}$.

Western blot. Total protein was extracted from cryopreserved sheep skin tissue using a total protein extraction kit. For SDS-PAGE electrophoresis, $200 \mu \mathrm{g}$ of the total protein sample was applied to each well. The separated proteins were transferred onto a nitrocellulose membrane, which was blocked with $5 \%$ skim milk at room temperature for $1 \mathrm{~h}$ and then incubated with a SLC45A2 rabbit polyclonal antibody (1:250 in TBST) and a GAPDH rabbit polyclonal antibody as the internal reference (1:1000 in TBST) at $4^{\circ}$ overnight. The membrane was then moved to room temperature for
$30 \mathrm{~min}$ the next day and washed 3 times in TBST for $10 \mathrm{~min}$ each. HRP-conjugated goat anti-rabbit IgG was then added to cover the membrane, followed by incubation at $37^{\circ}$ with horizontal shaking for $1 \mathrm{~h}$. The membrane was washed 6 times in TBST for 5 min each and then developed using the highly sensitive enhanced chemiluminescence solution (solution A:solution $\mathrm{B}=1: 1$ ) followed by film exposure. The resulting images were scanned. The SLC45A2 and GAPDH immunoblot results were analyzed using Image-ProPlus 6.0 software to measure the area and gray value for each target band. The target protein and the internal reference were compared for semi-quantitative analysis. Protein content $=$ area of the band $\times$ average gray scale; semi-quantitative target protein content $=$ target protein content/GAPDH protein content. All data are represented as the means \pm SEs. Univariate analysis of variance was performed using SPSS 19.0 software, and differences with $\mathrm{p}<0.05$ were considered statistically significant.

Immunohistochemistry. Paraffin-embedded tissues were sectioned, dewaxed for hydration, incubated in $3 \% \mathrm{H}_{2} \mathrm{O}_{2}$ at $37^{\circ}$ for $10 \mathrm{~min}$, and then washed 3 times in PBS with shaking (3 min each wash). Next, $5 \%$ goat serum was added dropwise to block the tissue, with incubation at $37^{\circ}$ for $20 \mathrm{~min}$. The excess liquid was then shaken off, and the SLC45A2 rabbit polyclonal antibody (1:100 in PBS) was added dropwise, with no treatment in the negative control. The samples were placed at $4^{\circ}$ overnight, rewarmed at $37^{\circ}$ for $30 \mathrm{~min}$ the next day and washed 3 times in PBS (3 min per wash). HRP-conjugated goat anti-rabbit secondary antibody working solution (1:150 in PBS) was then added dropwise, followed by incubation at $37^{\circ}$ for $30 \mathrm{~min}$ and 3 washes in PBS ( 3 min per wash). 5,5'-diaminobenzidine (DAB) was added to develop the staining for 1-10 min, followed by 3 final PBS washes (3 min each); after light hematoxylin counterstaining, dehydration, and clearing, the slices were mounted with neutral balsam and observed under a microscope.

Analysis of immunohistochemical data. Using DP software, $\geq 4$ fields of vision were selected on the hair follicle section for each coat color sample, and the collected images were analyzed with Image-ProPlus 6.0 software. The integrated optical density (IOD) values were measured, and the data are represented as the means \pm standard errors (SEM). 

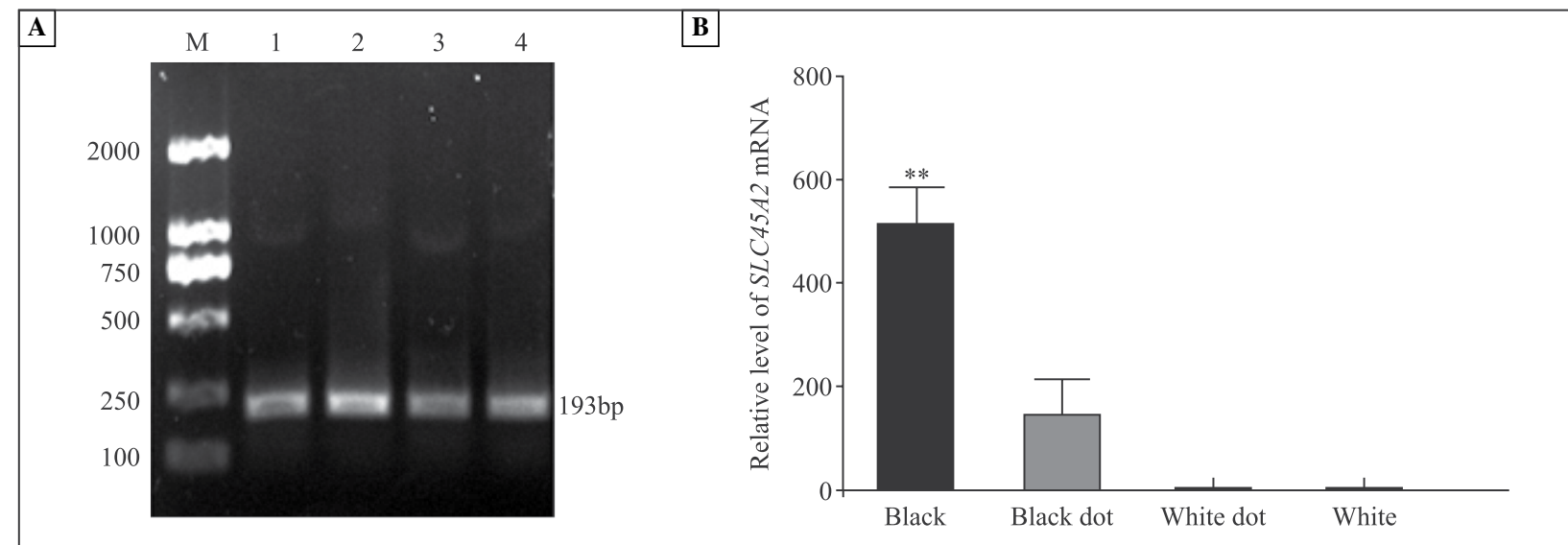

Figure 1. Analysis of $S L C 45 A 2$ gene expression in skin samples of sheep with different coat colors. A. Electrophoretic pattern of $S L C 45 A 2$ PCR products. M: DL 2000 DNA Marker; 1-4: SLC45A2 PCR products from black skin, black dots in piebald skin, white dots in piebald skin and white skin, respectively; B. QRT-PCR analysis of $S L C 45 A 2 \mathrm{mRNA}$ levels in sheep skin samples with different coat colors. ${ }^{*} \mathrm{P}<0.01 \mathrm{vs}$. other coat colors.

Statistical analysis. Univariate analysis of variance was performed using SPSS 19.0 software (IBM, Armonk, NY, USA). Data are represented as mean \pm standard error (SEM), differences with $\mathrm{p}<0.05$ were considered statistically significant.

\section{Results}

\section{Electrophoresis for PCR amplification}

By $1 \%$ agarose gel electrophoresis, the SLC45A2 PCR product was clear, with no nonspecific bands, and was determined to be approximately $193 \mathrm{bp}$ in length (Figure 1A). The target band on the gel was cut out and sent to BGI for sequencing. The correct sequence was obtained and confirmed, indicating that $S L C 45 A 2$ is expressed in normal sheep skin.

\section{QRT-PCR amplification}

The QRT-PCR results showed that the melting curves of the target and reference genes had only one clear peak. The $T_{m}$ value of the amplification products exhibited a high homogeneity, with no non-specific amplification or primer-dimers in the target or reference gene. In addition, the kinetics of SLC45A2 fluorescence quantitative amplification showed a clear "S" shape, with no obvious deviation peaks and a clear starting point of the peak (data not shown) which is in line with the requirements of a standard curve.

The QRT-PCR results further showed that the relative expression levels of SLC45A2 mRNA were $512.74 \pm 121.51$ in black skin, $143.38 \pm 119.31$ and $1.36 \pm 0.09$ in black dots and white dots of piebald skin, respectively, and $1.02 \pm 0.23$ in white skin (Figure $1 \mathrm{~B})$. The differences correspond to 377.0- and 502.7-fold increases in SLC45A2 mRNA in black skin compared with white dots of piebald skin and white skin, respectively. Furthermore, SLC45A2 expression in black dots of piebald skin was 105.4 and 140.6 times higher than that in white dots of piebald skin and white skin, respectively (Figure 1B).

\section{Western blot}

Western blot analysis showed that the total proteins from skin samples of sheep with different coat colors were positively immunoreactive to the SLC45A2 rabbit polyclonal antibody, with the target band located at the position of $58 \mathrm{kD}$ (Figure 2A). The protein band of a mass higher than $58 \mathrm{kD}$ in black sheep may represent phosphorylated protein. Protein band analysis showed that the relative expression levels of SLC45A2 protein were $0.85 \pm 0.17$ in black skin, $0.60 \pm 0.05$ and $0.34 \pm 0.07$ in black dots and white dots of piebald skin, respectively, and $0.20 \pm 0.05$ in white skin. These differences correspond to 2.5and 4.2-fold higher expression in black skin relative to white dots in piebald skin and white skin, respectively, and 1.8- and 3.0-fold higher expression in black dots in piebald skin compared with white dots in piebald skin and white skin, respectively. The differences between SLC45A2 protein expression in black skin and black dots of piebald skin were statistically significant (Figure 2B).

\section{Optical density analysis of the immunohistochemical demonstration of SLC-45A2 protein in skin sections of sheep with different coat colors}

The immunohistochemical staining demonstrated that SLC45A2 was expressed in dermal papilla, inner and outer root sheath, and hair follicle matrix in skin of sheep with different coat colors (Figure 3). 


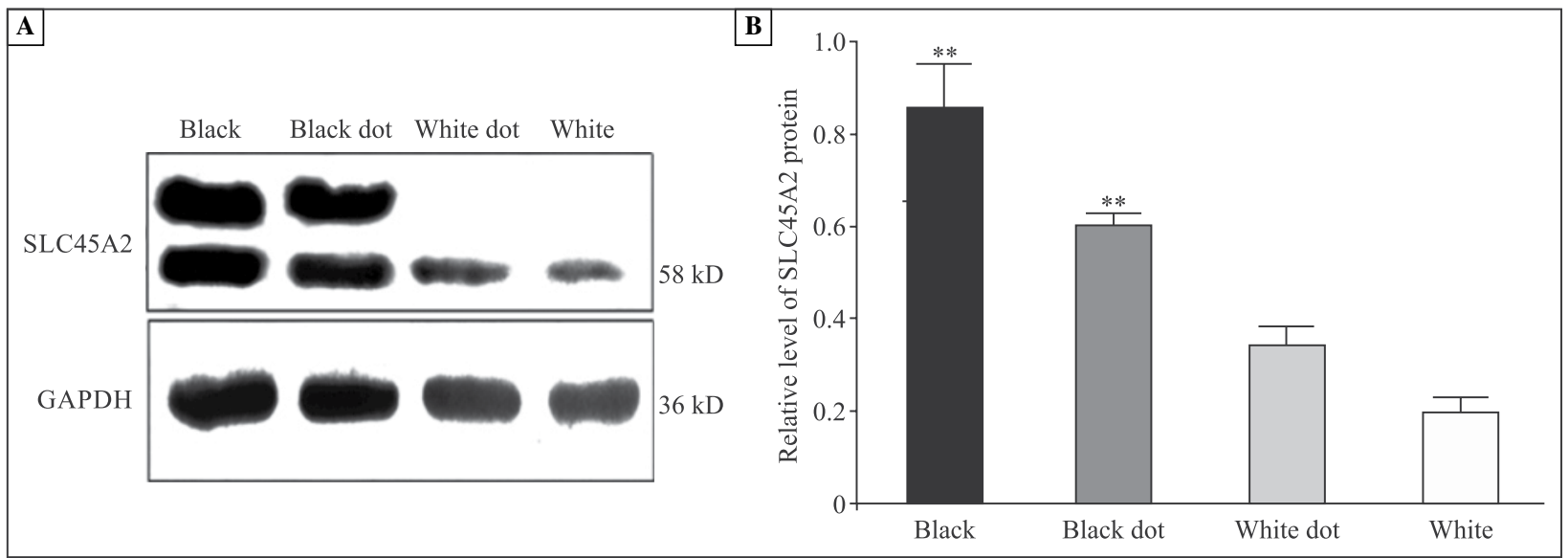

Figure 2. Western blot analysis of SLC45A2 protein expression in skin samples of sheep with different coat colors. A. The $58 \mathrm{kD}$ and $36 \mathrm{kD}$ bands were developed using a SLC45A2 polyclonal antibody and a GAPDH polyclonal antibody, respectively; B. Western blot analysis of SLC45A2 in the sheep skin of different coat colors. ${ }^{* *} \mathrm{P}<0.01 v s$. white dot piebald skin and white skin.

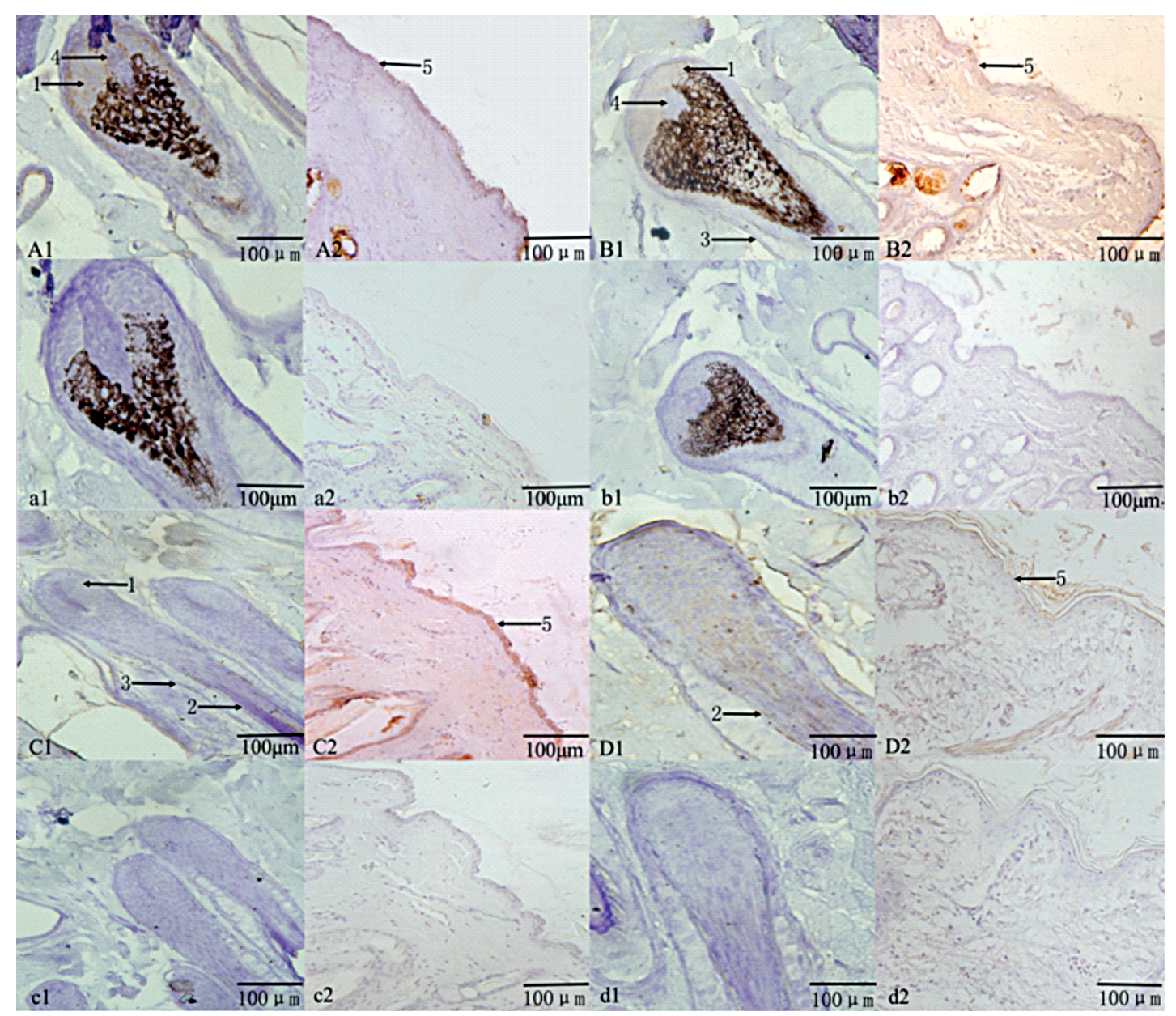

Figure 3. Distribution and expression of SLC45A2 protein in skin hair follicles of sheep with different coat colors. A1, a1, A2, a2: hair follicles in black skin; B1, b1, B2, b2: hair follicles in a black dot of piebald skin; C1, c1, C2, c2: hair follicles in a white dot of piebald skin; D1, d1, D2, d2: hair follicles in white skin. a1, a2, b1, b2: negative controls for black skin and black dots in piebald skin; c1, c2, d1, d2: negative controls for white dots in piebald skin and white skin, respectively. Sections of skin were stained by immunohistochemistry as described in Material and methods, negative controls were obtained by omitting primary antibody. Numbers denote: 1 - hair follicle matrix; 2 - inner root sheath; 3 - outer root sheath; 4 - dermal papilla; 5 - epidermis. 


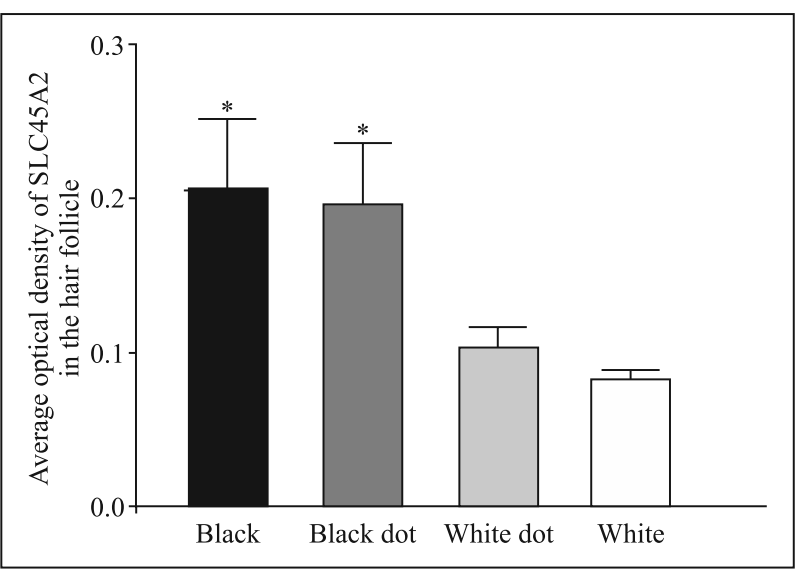

Figure 4. Average optical density analysis of SLC45A2 protein expression in hair follicles of sheep with different coat colors. Sections of skin were stained by immunohistochemistry and the analysis of staining's intensity was performed as described in Material and methods. ${ }^{*} \mathrm{P}<0.05$ vs. white dot piebald skin and white skin.

The average optical density (OD) values for SLC45A2 were $0.23 \pm 0.11$ in black skin, $0.19 \pm 0.09$ and $0.10 \pm 0.03$ in black dots and white dots of piebald skin, respectively, and $0.08 \pm 0.01$ in white skin. Thus, SLC45A2 is expressed at 2.3- and 2.9-fold higher levels in black skin compared with white dots in piebald skin and white skin, respectively, and 1.9- and 2.4-fold higher in black dots in piebald skin compared with white dots in piebald skin and white skin, respectively. The differences in the intensity of SLC45A2 protein immunoreactivity in black skin and black dots of piebald skin were statistically significant (Figure 4).

\section{Discussion}

Sheep is the most important animal for wool production. The diameter, length, and color of wool directly affect the economic value of the sheep, whose main traits are affected by both genetic factors $[20,21]$ and environmental conditions [22]. Mammalian dorsal hair color depends on the pheomelanin and eumelanin in melanosomes, with the relative contents of these two pigments determining the diversity of coat color [23]. Related studies showed that melanosomal proteins, membrane-associated transporter protein (MATP) and P protein, encoded by the MATP and OCA2 genes, and the melanocortin receptor (MC1R) help to determine the balance between pheomelanin and eumelanin in melanocytes [24, 25]. Fan et al. found that 49 known coat color genes are expressed in sheep skin, of which 13 genes were highly expressed in black sheep skin. These upregulated genes encode components of melanosomes and their precursors such as dopachrome tautomerase (DCT), MATP, tyrosinase (TYR), and tyrosinase related protein 1 (TYRP1) [26].

It was shown that SLC45A2 is associated with malignant melanoma (MM). This association has been unanimously validated in a series of pigmentation diseases and sunburn in children caused by dark skin, hair, eyes, and sun exposure [27]. Some alleles of SLC45A2 are associated with melanoma susceptibility in people with light skin [28]. SLC45A2 helps to regulate tyrosinase trafficking and $\mathrm{pH}$ maintenance in melanosomes [10, 29, 30]. MITF (Microphthalmia-associated transcription factor) is a transcription factor necessary for melanocyte development. MITF regulates the expression of the $S L C 45 A 2$ gene through the cAMP signaling pathway. Similar to the main pigmentation genes, MITF also regulates tyrosinase, TYRP1 and DCT [12]. SLC45A2 expression may also be affected by polymorphisms in the MITF promoter [31]. Huang et al. introduced a mutation in the structure of SLC45A2 protein (threonine 32 was replaced with serine, T32S) to mimic a human ortholog, potentially changing the function of the corresponding protein and further affecting the phenotype. SLC45A2 may also affect the development of melanocytes and cells of the enteric nervous system [32]. We designed our study based on the findings of previous investigations that suggested a link between SLC45-A2 and the formation of coat color $[2,10,26]$.

The relative expression of $S L C 45 A 2$ mRNA was significantly higher in black sheep skin than in white skin or in the black or white dots of piebald skin. The Western blot results were consistent with the mRNA expression levels, and positive expression of SLC45A2 was detected in the dermal papilla, the inner and outer hair root sheaths, and the hair follicle matrix in skin samples from sheep with different coat colors. A recent study showed that knockout of $S L C 45 A 2$ gene significantly reduced the $\mathrm{pH}$ of melanosomes, thereby reducing melanin content and tyrosinase activity [17]. These results suggest that the observed high expression of SLC45A2 in black skin and the black dots of piebald skin may be associated with the regulation of melanosome trafficking and tyrosinase activity, thereby influencing pigmentation. SLC45A2 may be localized to melanosomes. Melanin is synthesized and stored in these organelles and significantly affects their shape and color. Mutations in SLC45A2 cause the under-white (UW) phenotype in mice [2]. The melanosomes in these mice contain less melanin, and there are fewer mature organelles than in wild-type mice. The melanosomes of the mutant mice are small and 
crenated vs. round or oval in wild-type mice [33-35]. Because the human and mouse SLC45A2 genes are orthologous, this observation may provide primary clues into the possible function of the encoded protein $[35,36]$. Furthermore, mutations in SLC45A2 affect not only the catalytic activity of tyrosinase but also the secretion of other proteins in melanosomes during the II stage. For example, melanocytes from mice with the underwhite mutant phenotype were demonstrated to secrete vesicles containing tyrosinase, DCT and TYRP1 into the culture medium $[5,10]$. The latest study found that SLC-45A2 is involved in the intracellular trafficking of melanin/lysosomal components, which provides a basis for understanding how disruptions in the function of SLC45A2 result in the mistargeting of tyrosinase and hypopigmentation in OCA4 (Oculocutaneous albinism type 4) [37]. Our results, combined with the findings of previous studies, demonstrate that SLC45A2 can affect pigmentation by regulating the activity of melanin tyrosinase and the trafficking of melanosomes, indicating that SLC45A2 plays an important regulatory role in coat color pigmentation.

In summary, the relative mRNA and protein expression levels of SLC45A2 in skin samples of sheep with different coat colors suggest a correlation between SLC45A2 and the establishment of coat color, with increasing expression observed in order from white skin, white dots in piebald skin, black dots in piebald skin and black skin.

\section{Acknowledgements}

This work was supported by grants from the National Natural Science Foundation of China (31302049), the Doctoral Program of Higher Education (20131403120002), and Agro-Scientific Research in the Public Interest (201303119).

\section{References}

1. Harada M, Li YF, El-Gamil M, Rosenberg SA, Robbins PF. Use of an in vitro immunoselected tumor line to identify shared melanoma antigens recognized by HLA-A ${ }_{n} 0201$-restricted T cells. Cancer Res. 2001;61:1089-1094. PMID: 11221837.

2. Newton JM, Cohen-Barak O, Hagiwara $\mathrm{N}$ et al. Mutations in the human orthologue of the mouse underwhite gene $(\mathrm{uW})$ underlie a new form of oculocutaneous albinism, OCA4. Am J Hum Genet. 2001;69:981-988. doi: 10.1086/324340.

3. Inagaki K, Suzuki $\mathrm{T}$, Ito $\mathrm{S}$ et al. Oculocutaneous albinism type 4: six novel mutations in the membrane-associated transporter protein gene and their phenotypes. Pigment Cell Res. 2006;19:451-453. doi: 10.1111/j.1600-0749.2006.00332.x.

4. Vitavska O, Wieczorek H. The SLC45 gene family of putative sugar transporters. Mol Aspects Med. 2013;34:655-660. doi: 10.1016/j.mam.2012.05.014.

5. Costin GE, ValenciaJC, Vieira WD, LamoreuxML, Hearing VJ. Tyrosinase processing and intra-cellular trafficking is disrup- ted in mouse primary melanocytes carrying the underwhite (uW) mutation. A model for oculocutaneous albinism (OCA) type 4.J Cell Sci. 2003;116:3203-3212. doi: 10.1242/jcs.00598.

6. Yasushi T, Tamio S. Genetics of pigmentary disorders. Am J Med Genet Part C. 2004;131:75-81.

7. Hearing VJ. Biogenesis of pigment granules: a sensitive way to regulate melanocyte function.J Dermatol Sci. 2005;37:3-14. doi: 10.1016/j.jderm-sci.2004.08.014.

8. Kushimoto T, Valencia JC, Costin GE et al. The Seiji memorial lecture: the melanosome: an ideal model to study cellular differentiation. Pigment Cell Res. 2003;16:237-244. doi: 10.1034/j.1600-0749.2003.00034.x.

9. Fukamachi S, Shimada A, Shima A. Mutations in the gene encoding B, a novel transporter protein, reduce melanin content in medaka. Nat Genet. 2001;28:381-385. doi: 10.1038/ng584.

10. Du J, Fisher DE. Identification of aim-1 as the underwhite mouse mutant and its transcriptional regulation by MITF. J Biol Chem. 2002;277:402-406. doi: 10.1074/jbc.M110229200.

11. Mariat D, Taourit S, Guérin G. A mutation in the MATP gene causes the cream coat colour in the horse. Genet Sel Evol. 2003;35:119-133. doi: 10.1051/gse:2002039.

12. Gunnarsson U, Hellström AR, Tixier-Boichard M et al. Mutations in SLC45A2 cause plumage color variation in chicken and Japanese quail. Genetics. 2007;175:867-877. doi: 10.1534/ genetics.106.063107.

13. Nakayama K, Fukamachi S, Kimura H, Koda Y, Soemantri A, Ishida T. Distinctive distribution of AIM1 polymorphism among major human populations with different skin color. J Hum Genet. 2002;47:92-94. doi: 10.1007/s100380200007.

14. Graf J, Hodgson R, van Daal A. Single nucleotide polymorphisms in the MATP gene are associated with normal human pigmentation variation. Hum Mutat. 2005;25:278-284. doi: 10.1002/humu.20143.

15. Suzuki T, Tomita Y. Recent advances in genetic analyses of oculocutaneous albinism types 2 and 4. J Dermatol Sci. 2008;51:1-9. doi: 10.1016/j.jdermsci.2007.12.008.

16. Smith DR, Spaulding DT, Glenn HM, Fuller BB. The relationship between $\mathrm{Na}(+) / \mathrm{H}(+)$ exchanger expression and tyrosinase activity in human melanocytes. Exp Cell Res. 2004;298:521-534. doi: 10.1016/j.yexcr.2004.04.033.

17. Bin BH, Bhin J, Yang SH et al. Membrane-Associated Transporter Protein (MATP) regulates Melanosomal pH and influences tyrosinase activity. PLoS One. 2015;10:e0129273. doi: 10.1371/journal.pone.0129273.

18. Baxter LL, Pavan WJ. The oculocutaneous albinism type IV gene Matp is a new marker of pigment cell precursors during mouse embryonic development. Mech Dev. 2002;116:209-212. doi: 10.1016/S0925-4773(02)00130-2.

19. Nakayama A, Nguyen M T T, Chen C C, et al. Mutations in microphthalmia, the mouse homolog of the human deafness gene MITF, affect neuroepithelial and neural crest-derived melanocytes differently. Mech Dev. 1998;70:155-166. PMID: 9510032 .

20. Lamoreux ML, Wakamatsu K, Ito S. Interaction of major coat color gene functions in mice as studied by chemical analysis of eumelanin and pheomelanin. Pigment Cell Res. 2001;14:23-31. doi: 10.1034/j.1600-0749.2001.140105.x.

21. Bunge R, Thomas DL, Nash TG, Lupton CJ. Performance of hair breeds and prolific wool breeds of sheep in southern Illinois: wool production and fleece quality. J Anim Sci. 1996;74:25-30. PMID: 8778106.

22. Kidson SH, Fabian BC. The effect of temperature on tyrosinase activity in Himalayan mouse skin. J Exp Zool. 1981;215:91-97. doi: 10.1002/jez.1402150111. 
23. Thody AJ, Higgins EM, Wakamatsu K, Ito S, Burchill SA, Marks JM. Pheomelanin as well as eumelanin is present in human epidermis. J Invest Dermatol. 1991;97:340-344. doi: 10.1111/1523-1747.ep12480680.

24. Sturm RA, Teasdale RD, Box NF. Human pigmentation genes: identification, structure and consequences of polymorphic variation. Gene. 2001;277:49-62. doi: 10.1016/ S0378-1119(01)006 94-1.

25. Rees JL. Genetics of hair and skin color. Annu Rev Genet. 2003;37:67-90. doi: 10.1146/annurev.genet.37.110801.143233.

26. Fan R, Xie J, Bai J et al. Skin transcriptome profiles associated with coat color in sheep. BMC Genomics. 2013;14:1-12. doi: 10.1186/1471-2164-14-389.

27. Landi MT, Kanetsky P, Goldstein A et al. MC1R, ASIP, and DNA repair in sporadic and familial melanoma in a Mediterranean population. J Natl Cancer Inst. 2005:97;998-1007. doi: $10.1093 /$ jnci/dji176.

28. Fernandez LP, Milne RL, Pita G et al. SLC45A2: a novel malignant melanoma-associated gene. Hum Mutat. 2008;29:1161-1167. doi: 10.1002/hu-mu.20804.

29. Lucotte G, Mercier G, Diéterlen F, Yuasa I. A decreasing gradient of $374 \mathrm{~F}$ allele frequencies in the skin pigmentation gene SLC45A2, from the north of West Europe to North Africa. Biochem Genet. 2010;48:26-33. doi: 10.1007/s10528009-9289-4.

30. Dooley CM, Schwarz H, Mueller KP et al. SLC45A2 and $\mathrm{V}$-ATPase are regulators of melanosomal $\mathrm{pH}$ homeostasis in zebrafish, providing a mechanism for human pigment evolu- tion and disease. Pigment Cell Melanoma Res. 2013;26:205-217. doi: 10.1111/pcmr.12053.

31. Graf J, Voisey J, Hughes I, van Daal A. Promoter polymorphisms in the MATP (SLC45A2) gene are associated with normal human skin color variation. Hum Mutat. 2007;28:710-717. doi: 10.1002/humu.20504.

32. Huang J, Dang R, Torigoe D et al. Identification of genetic loci affecting the severity of symptoms of Hirschsprung disease in rats carrying Ednrbsl mutations by quantitative trait locus analysis. PLoS One. 2015;10:e0122068. doi: 10.1371/journal.pone.0122068.

33. Eicher EM, Green MC. The T6 translocation in the mouse: its use in trisomy mapping, centromere localization, and cytological identification of linkage group 3. Genetics. 1972;71:621-632. PMID: 5055128.

34. Davisson MT, Roderick TH, Akeson EC. The hairy ears (Eh) mutation is closely associated with a chromosomal rearrangement in mouse chromosome 15. Genet Res. 1990;6:167-178.

35. Sweet HO, Brilliant MH, Cook SA et al. A new allelic series for the underwhite gene on mouse chromosome 15. J Hered. 1998;89:546-551. PMID: 9864865.

36. Lehman AL, Silvers WK, Puri N, Wakamatsu K, Ito S, Brilliant $\mathrm{MH}$. The underwhite (uW) locus acts autonomously and reduces the production of melanin. J Invest Dermatol. 2000;115:601-606. doi: 10.1046/j.1523-1747.2000.00107.x.

37. Kondo T, Namiki T, Coelho SG, Valencia JC, Hearing VJ. Oculocutaneous albinism: developing novel antibodies targeting the proteins associated with OCA2 and OCA4.J Dermatol Sci. 2015;77:21-27. doi: 10.1016/j.jdermsci.2014.11.006.

Submitted: 22 February, 2016

Accepted after reviews: 22 July, 2016 Available as AoP: 16 August, 2016 